\title{
Estimating the Region of Attraction via Forward Reachable Sets
}

\author{
Ahmed El-Guindy, Dongkun Han, and Matthias Althoff
}

\begin{abstract}
We propose and implement an algorithm based on reachability analysis to estimate the region of attraction (ROA) of an equilibrium point for nonlinear systems. The stability region is obtained via the computation of forward reachable sets. We compare our results with well-established techniques in this area. In particular, we consider the optimization of the Lyapunov function (LF) sub-level set using sum-of-squares (SOS) decomposition, and the computation of backward reachable sets of a target set using the viscosity solution of a time-dependant Hamilton-Jacobi-Isaacs (HJI) formulation. Our method can overcome many limitations imposed on the applicability of Lyapunov-based approaches, such as conservatism in estimating the stability region, and difficulties associated with choosing a suitable LF. This is due to the fact that our reachability algorithm does not require a $L F$ in order to provide an estimate of the ROA. Various numerical examples show that our proposed approach can estimate the exact ROA quite accurately, and more importantly, scales moderately with the system dimension compared to alternative techniques.
\end{abstract}

\section{INTRODUCTION}

The estimation of stability regions of nonlinear systems is of fundamental importance in a wide range of applications, such as autonomous systems [1], [2], control of robotic manipulators [3], and transient stability of power systems [4], [5]. This problem is continuously receiving a lot of attention in literature as it still remains unsolved. Simply put, instead of examining stability of an equilibrium point, one is often more interested in determining the region from which an initial state can be attracted by this equilibrium. Finding a stability region, however, is difficult due to the fact that it is often a complicated set hard to be expressed analytically [6]. Developing efficient algorithms to tackle this problem is an extremely challenging task. These challenges consists of, but are not limited to, the conservatism in estimating the region of attraction (ROA), the overall computational costs, and the associated memory requirements, which grow rapidly with system dimension. In this work, we propose an algorithmic procedure based on forward reachability computations to provide accurate estimates of the stability region. A general literature review about reachability analysis is found in [7]-[9].

\section{A. Related Work}

The dominant techniques for estimating the ROA are based on Lyapunov's stability theory and its various extensions. These methods can offer sufficient conditions for verifying stability of ordinary differential equations (ODEs), using the so-called Lyapunov functions (LFs). Among its variations,

The authors are with the Institute of Robotics and Embedded Systems, Technische Universität München, 80333 Garching, Germany

E-mail: ahmed.elguindy@tum.de, dongkunh@umich.edu althoffein.tum.de the Zubov's method and the maximal LF method [10] can obtain the exact stability region. However, both techniques are generally difficult to employ, since they reformulate the problem into solving a partial differential equation (PDE) whose solution is not easily found in most cases. An alternative approach is based on the inner-approximation of the exact ROA via the optimization of the LF sub-level set. This approach proved to be quite effective following the rapid development of semi-algebraic geometry and sum of squares (SOS) decomposition, which resulted in increasingly efficient optimization techniques, involving linear matrix inequalities (LMIs) and semi-definite programming (SDP), see for example [11]-[16] and the references therein.

However, the Lyapunov-based techniques have several drawbacks which limit their applicability in practice. First, the approach relies on the existence of suitable LFs which are extremely difficult to find for nonlinear systems, due to the non-constructive nature of the Lyapunov theory; that is in other words the theorem only ensures the existence of a basin of attraction, yet it does not provide a systematic way to find an initial feasible LF. Although a quadratic LF can be easily constructed by solving the Lyapunov equation of the linearized system, it only captures the local behaviour of the nonlinear ODEs around the equilibrium point.

Additionally, Lyapunov methods suffer from conservatism in estimating the ROA, since the techniques often relax the optimization problem, to maximize the sub-level set of the LF. This is done either by enforcing convexity of the solution with conservative LMI conditions, or by employing non-convex bilinear matrix inequalities [17]. Another disadvantage of this class of techniques is that it cannot formally verify if system constraints are met, e.g. if a robot manipulator exceeds maximum torque or when a bus voltage of a power system drops beyond limitations imposed by the grid operator. This is due to the fact that Lyapunov methods only analyze if a steady state of a disturbed system is eventually reached.

An alternative direction is a class of methods which is not based on the Lyapunov stability theory. An overview covering the early contributions of this kind of techniques is found in [18]. Very recently, the computation of backward reachable enclosures using level set methods (LSMs), starting from a small neighborhood around the equilibrium point, has turned out to be an effective tool that can provide accurate estimates of the ROA, see e.g. [19]. This is achieved using an Eulerian technique via the formulation of a Hamilton-Jacobi-Isaacs (HJI) PDE, where it is proven that the viscosity solution of the time-dependent HJI PDE provides an implicit surface representation of the continuous backward reachable set [20]. The main drawback of this 
technique, however, is that the computational requirements grow rapidly with the system dimension, due to the fact that no analytical solution exists for the set of PDEs. Thus, one has to continuously perform a discretization of the state space, resulting in an exponential complexity with respect to the number of continuous state variables. Another limitation of LSMs is that they only provide an accurate approximation of the reachable set, rather than a rigorous enclosure of it; thus it does not qualify as a formal technique for the estimation of the ROA [21].

The approach presented in this work is similar in spirit to the aforementioned class of methods; however, the key distinction is that our algorithm is based on Lagrangian techniques, which in contrast to Eulerian methods, compute reachable sets similar to numerical integration. This is achieved by propagating the set of reachable states instead of only computing the solution for a single value. The consequence of this is that we can handle higher-dimensional systems, since the associated memory requirements grow moderately with the system dimension, see e.g. [22], [23].

\section{B. Contributions}

To the best of our knowledge, this is the first work presenting an algorithmic procedure to estimate the ROA of an equilibrium point via the computation of forward reachable sets. In this work, we describe and implement a scalable and versatile algorithm that can provide accurate, and more importantly, provable estimates of the stability region. By versatile, we refer to the ability of the algorithm to deal with general systems involving non-polynomial models, thus covering a wide range of applications. Furthermore, this is the first work comparing three different techniques that can provide estimates of the stability region. Namely, we compare our Lagrangian approach (forward reachability computation) with the Eulerian method (backward reachability computation) and the Lyapunov direct method (optimization of the LF sub-level set).

The remainder of the paper is organized as follows: In Section II we formulate the problem and present basics for computing reachable sets using the Lagrangian technique. The estimation algorithm is described in Section III, and in Section IV we illustrate the applicability of our algorithm and compare it with existing techniques. Conclusions and future work are discussed in Section V.

\section{PREliminaries}

We denote by $\mathbb{N}^{n}, \mathbb{R}^{n}$ the set of natural and real numbers with dimension $n$. For two sets $\mathcal{X}, \mathcal{Y} \subset \mathbb{R}^{n}$, the operator $\oplus$ returns the Minkowski sum $\mathcal{X} \oplus \mathcal{Y}=\{x+y \mid x \in \mathcal{X}, y \in$ $\mathcal{Y}\}$. For two real numbers $\underline{a}, \bar{a} \in \mathbb{R}^{n}$ with $\underline{a}<\bar{a}$, a closed multidimensional interval is $\mathcal{I}=[\underline{a}, \bar{a}]$, and the union of $k$ intervals is denoted by $\cup_{i=1}^{k} \mathcal{I}_{i}$.

\section{A. Problem Formulation}

Consider autonomous nonlinear systems of the form

$$
\dot{x}(t)=f(x(t)),
$$

where $x \mapsto \mathbb{R}^{n}$ is the vector of state variables, and the function $f: \mathbb{R}^{n} \mapsto \mathbb{R}^{n}$ is locally Lipschitz continuous. The dependency on the time $t$ is often omitted for simplicity of notation. Our objective is to estimate the stability region

$$
\mathcal{S}^{e}\left(x_{s}\right)=\left\{x(0) \in \mathbb{R}^{n}: \lim _{t \rightarrow \infty} \gamma(x ; t)=x_{s}\right\},
$$

where $x_{s} \in \mathbb{R}^{n}$ is the stable equilibrium point (SEP) and $\gamma(x ; t)$ is a system trajectory of (1) starting from the initial condition $x(0)$.

The problem is approached in this paper using reachability analysis, which has proven to be an efficient tool for safety verification in a variety of applications, such as e.g. aircrafts collision avoidance [20], power systems [24], [25], and robotic aerial vehicles [26]. Reachability analysis basically determines the set of states that a system can reach, over a time horizon $t_{f}$, starting from a set of initial states $\mathcal{R}(0)$ :

$$
\begin{aligned}
\operatorname{reach}\left(\mathcal{R}(0), t_{f}\right)= & \left\{x(t) \in \mathbb{R}^{n}: x(t) \text { satisfies }(1)\right. \\
& \text { within } \left.\left[0, t_{f}\right] \text { for } x(0) \in \mathcal{R}(0)\right\}
\end{aligned}
$$

Note that (3) corresponds to the exact reachable set. However, it is proven that exact reachable sets for the class of nonlinear systems are not computable [27], thus an over-approximation, which includes all behaviours of (1) is performed as tightly as possible.

\section{B. Computation of Forward Reachable Sets}

Our reachability algorithm is based on abstracting the original differential equations (1) into a linear differential inclusion (LDI) for each consecutive time interval $\tau_{k}:=$ $\left[t_{k}, t_{k+1}\right]$, where $t_{k}=k \cdot r$, such that $k \in \mathbb{N}$, and $r \in$ $\mathbb{R}^{+}$corresponds to the time step and the time increment, respectively. Since the linearization of (1) causes additional errors, these errors are determined in an over-approximated manner and considered as uncertain inputs. Recomputing the linearization for each $\tau_{k}$, allows the over-approximation to remain small and ensures accurate results.

After introducing the linearization point $\tilde{x}_{k} \in \mathbb{R}^{n}$, with $k$ as the time step, and defining $\Delta x:=x-\tilde{x}$, we abstract the original nonlinear system (1) at each time interval $\tau_{k}$, by a LDI expressed as a first order Taylor expansion around $\tilde{x}_{k}$ with the Lagrangian remainder:

$$
\begin{aligned}
\forall t & \in \tau_{k}=\left[t_{k}, t_{k+1}\right]: \\
\dot{x}(t) & \in \underbrace{\left.\sum_{j=1}^{n} \frac{\partial f(x)}{\partial x_{j}}\right|_{x=\tilde{x}_{k}} \Delta x_{j}}_{=: A_{k} \Delta x} \oplus \underbrace{f\left(\tilde{x}_{k}\right) \oplus \mathcal{L}\left(\tau_{k}\right)}_{=: \mathcal{U}\left(\tau_{k}\right)},
\end{aligned}
$$

where $A_{k} \in \mathbb{R}^{n \times n}$ is the system matrix, $\mathcal{U}$ is the set of uncertain inputs, and $\mathcal{L}$ denotes the set of the Lagrangian remainder containing all possible linearization errors within the time interval $\tau_{k}$. It can be over-approximated according to $[28]$ as

$$
\begin{aligned}
\mathcal{L}\left(\tau_{k}\right)=\left\{L \in \mathbb{R}^{n}: L_{j}\right. & =1 /{ }_{2} \Delta x^{T} H^{(j)}(\zeta) \Delta x, \\
x & \left.\in \mathcal{R}\left(\tau_{k}\right), \zeta \in \mathbf{I H}\left(\mathcal{R}\left(\tau_{k}\right)\right)\right\},
\end{aligned}
$$


with $\mathbf{I H}(\cdot)$ returning the interval enclosure of a set and $H^{(j)}(\zeta), j \in\{1 \ldots n\}$ is the set of Hessian matrices, corresponding to the second-order partial derivatives of the function $f(\cdot)$ :

$$
H^{(j)}(\zeta):=\left[\begin{array}{ccc}
\left.\frac{\partial^{2} f_{j}(x)}{\partial x_{1}^{2}}\right|_{x=\zeta} & \cdots & \left.\frac{\partial^{2} f_{j}(x)}{\partial x_{1} \partial x_{n}}\right|_{x=\zeta} \\
\vdots & \ddots & \vdots \\
\left.\frac{\partial^{2} f_{j}(x)}{\partial x_{n} \partial x_{1}}\right|_{x=\zeta} & \cdots & \left.\frac{\partial^{2} f_{j}(x)}{\partial x_{n}^{2}}\right|_{x=\zeta}
\end{array}\right],
$$

with the subscript $j$ corresponding to the $j$-th coordinate.

Here $\mathcal{R}\left(\tau_{k}\right)$ denotes the reachable set at $\tau_{k}$ which is computed shortly after. We define $r:=t_{k+1}-t_{k}$ and $u_{c}$ as the center of $\mathcal{U}$. The reachable set $\mathcal{R}_{a}\left(t_{k+1}\right)$ of the dynamics $\dot{x}=A_{k} \Delta x+u_{c}$, enclosed by the LDI (4), is based on the well-known solution of linear state-space equations

$$
\mathcal{R}_{a}\left(t_{k+1}\right)=e^{A_{k} r} \mathcal{R}\left(t_{k}\right) \oplus \mathcal{R}_{p}(r),
$$

where $\mathcal{R}_{p}(r)$ is the set which over-approximates the particular solution of the linear state-space equation. The reachable set at the next point in time $t_{k+1}$, and for the time interval $\tau_{k}=\left[t_{k}, t_{k+1}\right]$, are obtained as follows

$$
\begin{aligned}
\mathcal{R}\left(t_{k+1}\right) & =\mathcal{R}_{a}\left(t_{k+1}\right) \oplus \mathcal{R}_{u}(r), \\
\mathcal{R}\left(\tau_{k}\right) & =\operatorname{conv}\left(\mathcal{R}\left(t_{k}\right), \mathcal{R}_{a}\left(t_{k+1}\right)\right) \oplus \mathcal{F} \oplus \mathcal{R}_{u}(r) .
\end{aligned}
$$

The operator $\operatorname{conv}(\cdot)$ returns the convex enclosure of two sets, and the set $\mathcal{F}$ considers enlargement of the resulting convex hull enclosure, in order to account for the assumption that trajectories over $\tau_{k}$ are straight lines. Finally the reachable set $\mathcal{R}_{u}(r)$ accounts for the uncertainty of the input set $\mathcal{U}$. The expressions of the following sets: $\mathcal{L}, \mathcal{R}_{p}(r), \mathcal{F}$, and $\mathcal{R}_{u}(r)$ are derived in our previous work [9, Ch. 3].

\section{ESTIMATION OF THE ROA}

In this section, we present our proposed algorithm to estimate the stability region of the equilibrium point $x_{s}$ using forward reachability computations. An overview covering the basic steps of our approach is illustrated in Fig. 1. The estimation algorithm consists of the following steps:

Step (1) Construction of a target set: Since we require a termination condition for forward reachable sets, we establish a small provable region around the equilibrium point. Once a reachable set is enclosed by this region, we can terminate our computations and conclude that all solutions converge to the equilibrium point. To this end, we introduce the target set $\mathcal{T}_{g} \subset \mathbb{R}^{n}$ defined as the multidimensional interval

$$
\begin{aligned}
\mathcal{T}_{g} & =\left[\underline{x}_{s}, \bar{x}_{s}\right], \\
\text { with } \quad \underline{x}_{s} & =x_{s}-\epsilon, \quad \bar{x}_{s}=x_{s}+\epsilon,
\end{aligned}
$$

where $\epsilon$ is chosen to be sufficiently small (e.g. 1E-3) such that $\mathcal{T}_{g}$ only surrounds a small neighborhood around $x_{s}$, thus ensuring attraction to the equilibrium. This step is based on the definition of stability in the sense of Lyapunov [6, Def. 4.1], which states that $x_{s}$ attracts any initial states $x(0)$ located adequately close.

Step (2) Enlargement of the target set: The step is illustrated in Fig. 2 by the blue boxes. Here we perform an

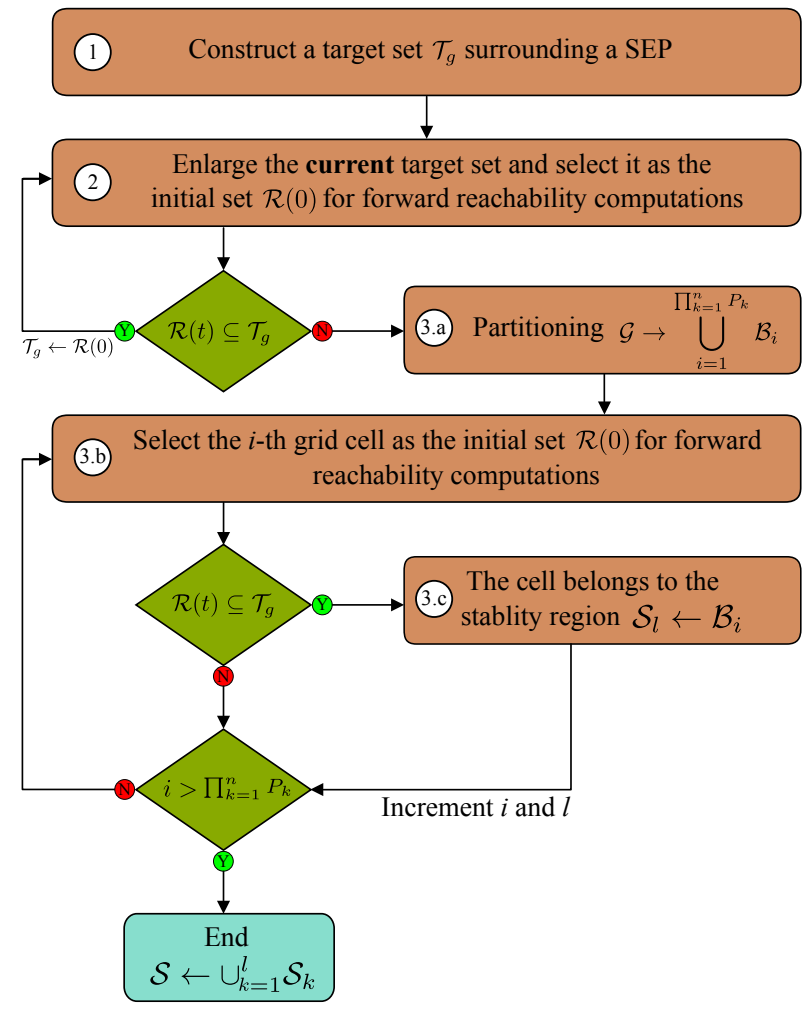

Fig. 1. Overview of our proposed approach to estimate the ROA of an equilibrium point via the computation of forward reachable sets.

iterative procedure to enlarge $\mathcal{T}_{g}$ using forward reachability computations. The enlargement of $\mathcal{T}_{g}$ is executed for two reasons: Firstly, in order to obtain a larger provable region that guarantees convergence to the equilibrium; secondly, and more importantly, to reduce the overall computational costs associated with reachability computations of initial states far away from $\mathcal{T}_{g}$, as described shortly.

In each iteration, the enlarged target set is selected as the initial reachable set, such that

$$
\mathcal{R}(0)=\lambda \cdot\left[\underline{x}_{s}, \bar{x}_{s}\right],
$$

where $\lambda \in \mathbb{R}^{+}$is a tunable enlargement factor set by the user. For practical reasons, the iterative procedure stops if

$$
\mathcal{L} \nsubseteq \overline{\mathcal{L}}
$$

where the set $\overline{\mathcal{L}}$ is the maximum linearization errors chosen by the user. Therefore, the enlargement procedure of $\mathcal{T}_{g}$ is limited and would only result in a very conservative stability region using forward reachability computations. Hence, in the following step, we investigate the domain surrounding $x_{s}$ using a complementary procedure in order to estimate the ROA more accurately.

Step (3) Partitioning of the domain of investigation: The step is illustrated in Fig. 2, where the black boxes present a recursive discretization of the grid. Here, we perform a partitioning of the state space to overcome the limitations imposed on the enlargement of $\mathcal{T}_{g}$. Clearly, it is neither feasible nor practical to discretize the whole state space when attempting to estimate the stability region. This is based on 


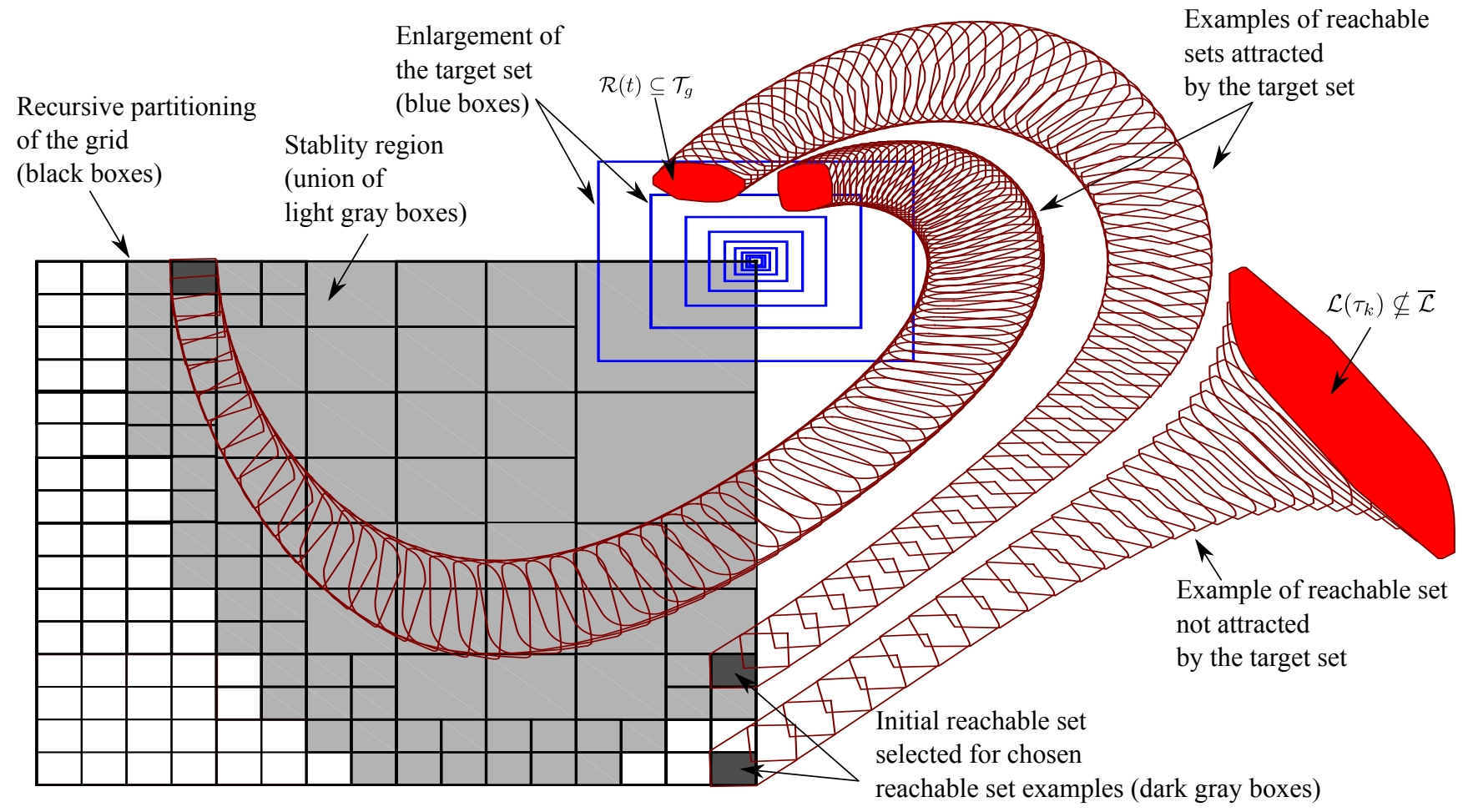

Fig. 2. Step-by-step computation of the stability region using forward reachable sets. The blue boxes represent the iterative enlargement of the target set $\mathcal{T}_{g}$ constructed around a SEP. The boxes show the recursive partitioning of the grid to provide an accurate estimate of the ROA. The dark gray areas are the cells whose reachable set is attracted by the enlarged target set, whereas the white areas correspond to the cells whose reachable set does not converge. Three random cells were chosen to illustrate the evolution of the reachable set (dark red), where the computation stops (highlighted by the red area) when the reachable set $\mathcal{R}(t), t \in\left[0, \infty\right.$ [ is either a subset of the target set $\mathcal{R}(t) \subseteq \mathcal{T}_{g}$ or if the Lagrangian remainder is not a subset of the maximum linearization errors $\mathcal{L} \nsubseteq \overline{\mathcal{L}}$ (see (12)).

the fact that one is particularly interested in a specific domain of investigation around the equilibrium point. We denote the domain of investigation by $\mathcal{G} \subset \mathbb{R}^{n}$, which we partition into

$$
\operatorname{partition}(\mathcal{G}, P)=\cup_{i=1}^{\sigma} \mathcal{B}_{i}, \quad \sigma=\prod_{k=1}^{n} P_{k},
$$

where $\mathcal{B}_{i} \subset \mathbb{R}^{n}, i \in\left\{1 \ldots \prod_{k=1}^{n} P_{k}\right\}$ are the resulting grid cells, and $P \in \mathbb{N}^{n}$ is the vector specifying the partitioning size in each dimension, with the subscript $k$ denoting the $k$-th element of the vector $P$.

Following the discretization of $\mathcal{G}$, each grid cell is selected as the initial set for the reachability algorithm. The cell is formally proven to belong to the stability region of the equilibrium point if the resulting reachable set of states is a subset of the target region, i.e $\exists t: \mathcal{R}(t) \subseteq \mathcal{T}_{g}$. During the implementation of our algorithm, we have found that a recursive partitioning of the grid, starting with a large cell size is more efficient in terms of computational time than the discretization of the domain of investigation directly with a fixed $P$. This is because a recursive partitioning with different sizes allows one to rapidly explore large areas around $\mathcal{T}_{g}$, and if these areas converge to the equilibrium, one does not need to re-examine them.

Step (4) Aggregation of results: Following the examination of all cells, the stability region is defined by the union of cells formally proven to converge to $\mathcal{T}_{g}$, that is

$$
\begin{aligned}
\mathcal{S}\left(x_{s}\right) & :=\cup_{i=1}^{l} \mathcal{B}_{i} \subset \mathcal{S}^{e}\left(x_{s}\right), \\
\text { s.t : } \quad \exists t: \operatorname{reach}\left(\mathcal{B}_{i}, t\right) & \subseteq \mathcal{T}_{g} .
\end{aligned}
$$

The overall procedure to obtain the stability region is summarized in Algorithm 1 and Algorithm 2, which outlines the computation of reachable sets and the estimation of the ROA, respectively. Six parameters are passed to the algorithm by the user: the stable equilibrium point $x_{s}$, the domain of investigation $\mathcal{G}$, the target set enlargement factor $\lambda$, the partitioning size of the grid $P$, the time increment $r$, and the maximum linearization errors $\overline{\mathcal{L}}$.

\section{RESUlTS}

We demonstrate the applicability of our proposed algorithm on various benchmark examples. All computations are performed in MATLAB2014b on a standard computer. Our algorithm computes forward reachable sets using the CORA toolbox [29]. Our results are compared with other wellestablished tools. Namely, we consider the level set (LST) [30] and the SMRSOFT [31] toolboxes, which compute the ROA using alternatives techniques.

In particular, LST computes the backward reachable set of a target set enclosing an equilibrium point using the viscosity solution of a time-dependant HJI PDE, while SMRSOFT estimates the stability region by maximizing the LF sub-level 
set using SOS programming. In this paper, the LF $V(x)$ is usually chosen to be quadratic in the form of

$$
V(x)=x^{T} M x .
$$

Here $M \in \mathbb{R}^{n \times n}$ is a real symmetric matrix obtained by solving the continuous Lyapunov equation $A^{T} M+M A+Q=0$, where $Q$ is chosen to be the identity matrix, and $A$ is the system matrix of the linearized system at $x_{s}$. Note that we are only using quadratic LFs.

\section{A. Two-Dimensional Examples}

First, we consider the Van-der-Pol oscillator

$$
\begin{aligned}
& \dot{x}_{1}=-x_{2}, \\
& \dot{x}_{2}=-x_{2}\left(1-x_{1}^{2}\right)+x_{1} .
\end{aligned}
$$

The system has a stable equilibrium point at the origin, the domain of investigation is defined by the multidimensional interval $\mathcal{G}:=[-2.5,2.5] \times[-2,2]$, where the operator $\times$ denotes the Cartesian product, and the chosen LF is $V(x)=$ $1.5 x_{1}^{2}-x_{1} x_{2}+x_{2}^{2}$ (only needed in SMRSOFT). The estimated ROA is shown in Fig. 3. The regions are obtained using our proposed algorithm, LST, and SMRSOFT. The gray areas show the cells attracted by the equilibrium, following the recursive partitioning of the grid. Note that additional partitioning takes place when examining areas far away from the equilibrium point (target set). It can be seen that the estimate of the stability region using the Lyapunovbased approach is relatively conservative compared to both

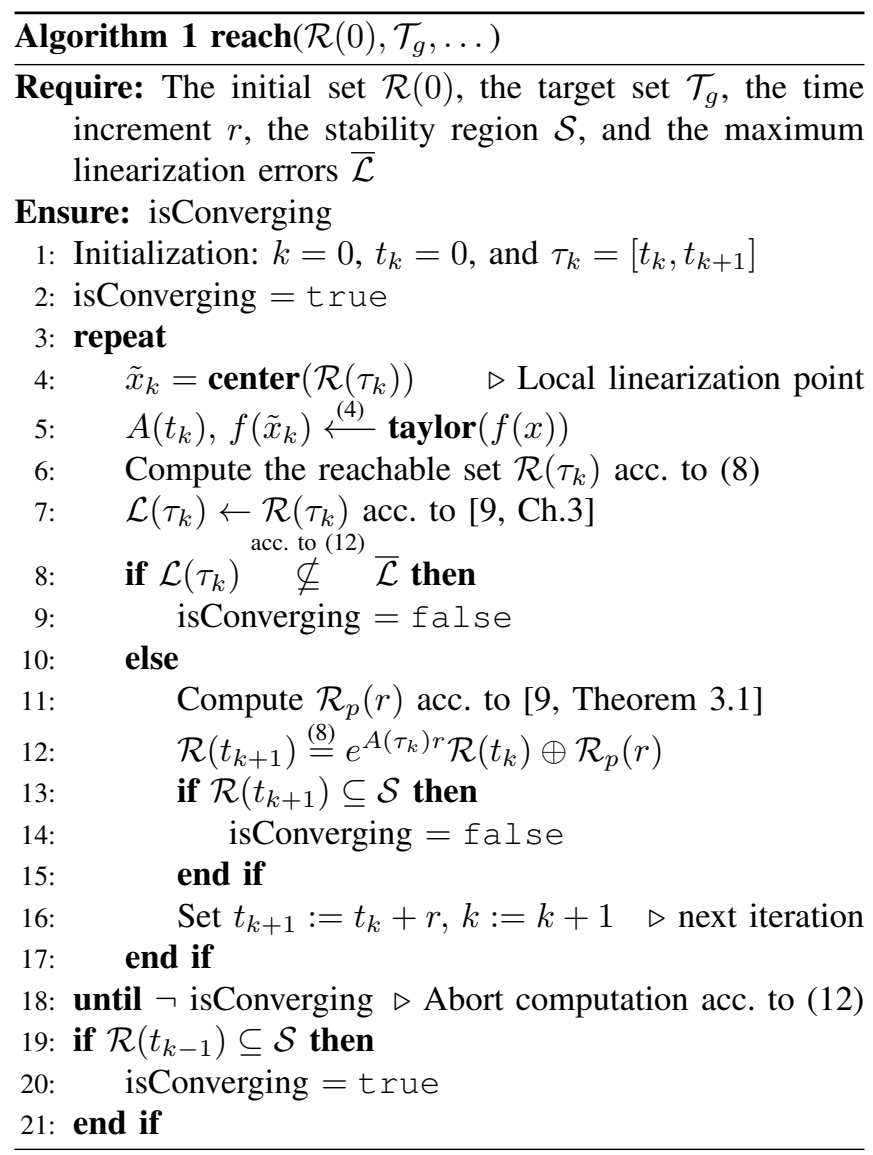

regions obtained by forward/backward reachable sets, and it is slightly more accurate using LST.

Next, we examine the following example from [32]:

$$
\begin{aligned}
& \dot{x}=-2 x+y+x^{3}+y^{5}, \\
& \dot{y}=-x-y+x^{2} y^{5} .
\end{aligned}
$$

The system has a stable equilibrium point at the origin. Here, the domain of investigation is $\mathcal{G}:=[-2,2] \times[-2,2]$. The stability region is provided in Fig. 4, where it can be seen that both forward/backward reachability algorithms still provide larger estimates of the ROA compared to the ellipsoid associated with the sub-level set of the chosen LF $V(x)=5 / 18 x_{1}^{2}-1 / 9 x_{1} x_{2}+4 / 9 x_{2}^{2}$.

Finally, we examine the single-machine-infinite-bus (SMIB) system. It is a benchmark example frequently used for analysis of power systems. The system is modelled by

$$
\begin{aligned}
\dot{\delta} & =\omega, \\
\dot{\omega} & =\frac{1}{H}\left(P_{m}-P_{e} \sin (\delta)-D \omega\right),
\end{aligned}
$$

where the state variables $\delta$ and $\omega$ correspond to the generator angle and its rotational speed. The meaning and values of the constant parameters $H, P_{e}, P_{m}$, and $D$ can be obtained from [33, Ch. 12]. The equilibrium point is $x_{s}=[0.835,0]$, the domain of investigation is $\mathcal{G}:=[-0.5,2.5] \times[-3,3]$, and the chosen LF is $V(x)=3.854 x_{1}^{2}+0.313 x_{1} x_{2}+0.142 x_{2}^{2}+$ $0.019 x_{2}$. The ROA is illustrated in Fig. 5, where both reach-

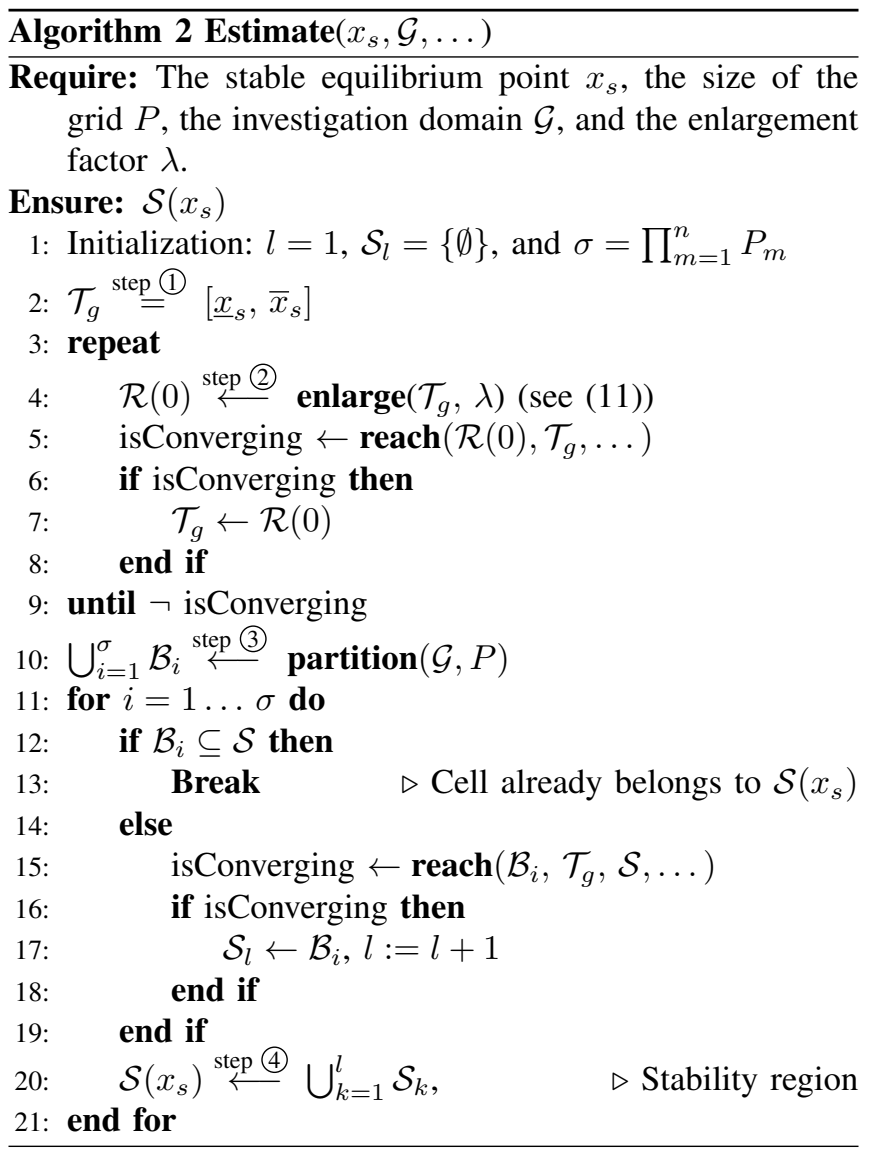


ability algorithms significantly outperform the Lyapunovbased method in terms of accuracy. Here, additional conservatism can be observed by the estimate of the sub-level set of the LF, due the fact that SMRSOFT can only work with systems with a polynomial vector field; thus, important system information was lost during the polynomialization of the nonlinear system. This step is not required using our algorithm as it fully considers the system nonlinearities.

\section{B. High-Dimensional System}

The proposed algorithm also works for higher dimensions. The first example is a 3-dimensional system with a polynomial vector from [34]:

$$
\begin{aligned}
& \dot{x}_{1}=-x_{2}, \\
& \dot{x}_{2}=-x_{3}, \\
& \dot{x}_{3}=-x_{1}-2 x_{2}-x_{3}+x_{1}^{3} .
\end{aligned}
$$

The system has a stable equilibrium point at the origin, the chosen domain of investigation is $\mathcal{G}:=[-1,1] \times[-2,2] \times$ $[-2,2]$, and the stability region is provided in Fig. 6. In this example, it is clear that the accuracy of the stability region is better using our algorithm compared to backward reachability computations. Note that we could not visualize the estimate of the ROA using the sub-level set of the LF due to some unknown problems with the toolbox SMRSOFT, although numerous LFs were examined.

The final example is a 4-dimensional non-polynomial system. Here we consider the inverted pendulum on a moving cart governed by the differential equations

$$
\begin{aligned}
\left(m_{c}+m_{p}\right) \ddot{x} & =u+m_{p} \ddot{\theta} \cos (\theta)-m_{p} l \dot{\theta}^{2} \sin (\theta), \\
l \ddot{\theta}-\ddot{x} \cos (\theta) & =g \sin (\theta),
\end{aligned}
$$

where $m_{c}, m_{p}$ are the masses of the cart and the pendulum, respectively, $l$ is the length of the pendulum, $g$ is the gravitational acceleration, and $u$ is the force applied to the

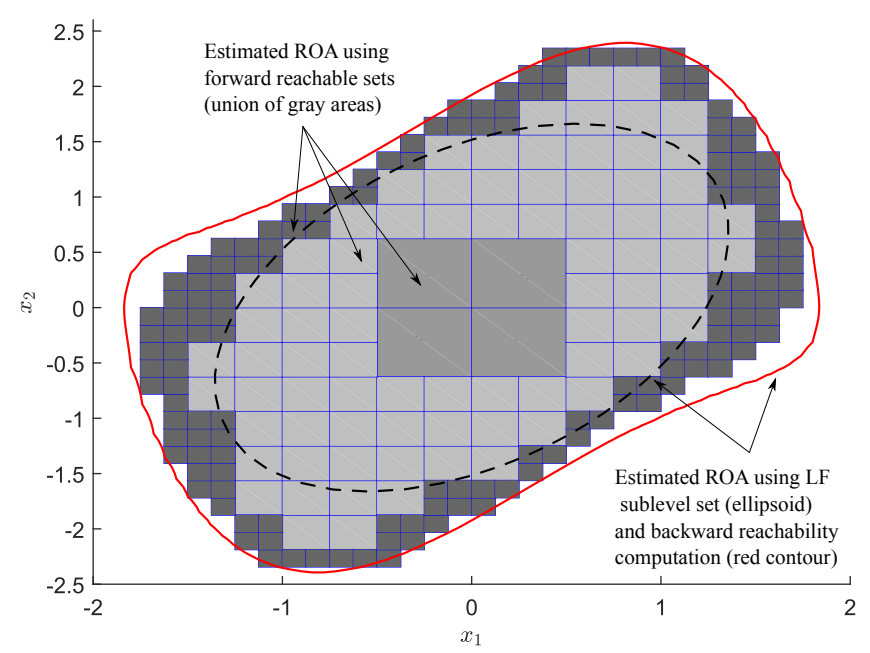

Fig. 3. Estimation of the stability region of (16) using our proposed algorithm (gray areas), LST (red-solid contour), and SMRSOFT (blackdotted ellipsoid). The equilibrium point is located at the origin. cart. The state variables are the cart position $x$, its velocity $\dot{x}$, the pendulum angle $\theta$, and its rotational speed $\dot{\theta}$. The system is controlled using a linear-quadratic regulator (LQR) to stabilize the pendulum in the upright position $\theta=0$.

In this example, we would like to identify the critical pendulum angle from which the LQR would manage to bring the pendulum to equilibrium. Based on the practical constraints of the chosen setup, the domain of investigation can be chosen as $\mathcal{G}:=[-0.5,0.5] \times[-1,1] \times[-\pi / 2, \pi / 2] \times$ $[-2,2]$. Fig. 7 shows a projection of the ROA obtained using our reachability algorithm, which can be easily verified either practically or using numerical simulations at the boundaries of the estimated stability region. We could not visualize the ROA using LST, as the algorithm exceeded the maximum associated memory (16GB) of our machine at $t=157 \mathrm{~s}$; thus, we had to abort the computation in MATLAB. Additionally, similar to the 3-D example, we did not manage to obtain an estimate of the ROA using SMRSOFT, although numerous LFs were examined.

\section{Discussion}

It is shown in Fig. 3 to Fig. 6 that both reachability algorithms provide fairly accurate and identical estimates of the stability region compared to the conservative ROA estimated using the sub-level set of the LF. The computational time required to estimate the ROA for each example using our algorithm is listed in Table I. It can be seen that our reachability algorithm clearly scales moderately; however, the associated memory requirements grew rapidly for backward reachable computations as seen in the 4-D example (20). This is due the fact that the computation of backward reachable sets requires a continuous partitioning of the grid, in order to find the viscosity solution of the HJIPDE formulation, i.e. exponential complexity with respect to the number of state variables. Although our algorithm suffers from an exponential complexity as well, this complexity is

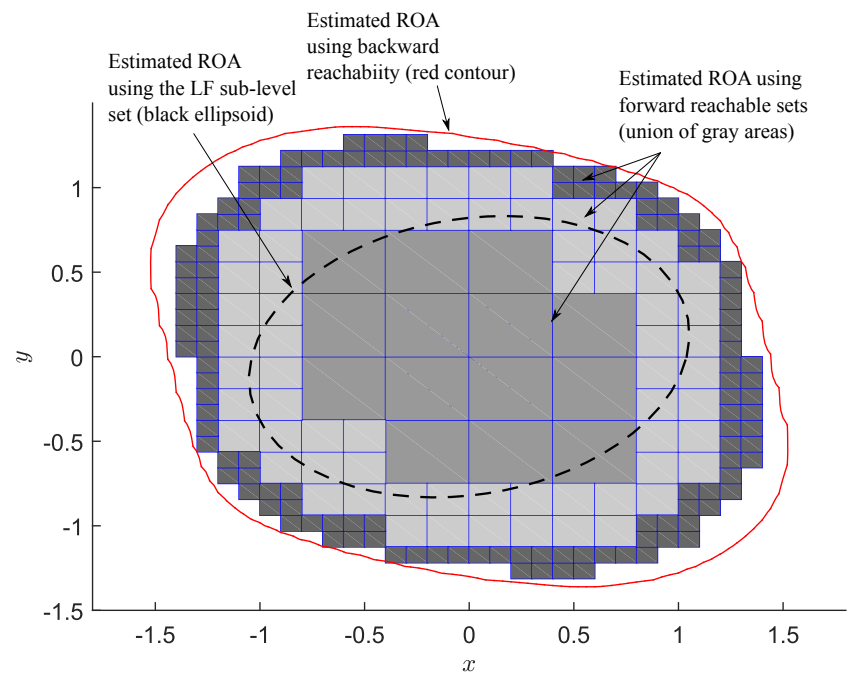

Fig. 4. Estimation of the stability region of (17) using our proposed algorithm (gray areas), LST (red-solid contour), and SMRSOFT (blackdotted ellipsoid). The equilibrium point is located at the origin. 


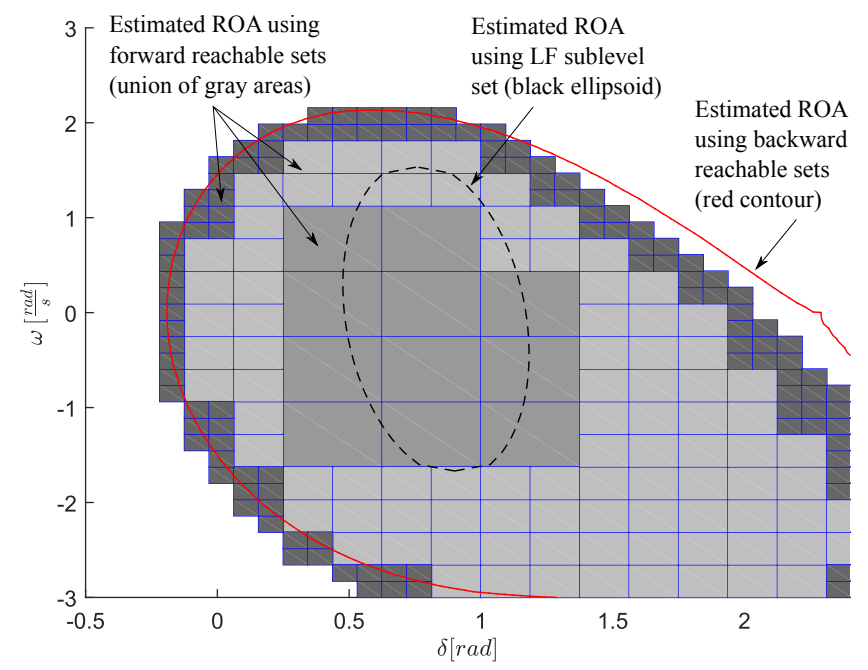

Fig. 5. Estimation of the stability region of (18) using our proposed algorithm (gray areas), LST (red-solid contour), and SMRSOFT (blackdotted ellipsoid). The equilibrium point is located at $x_{s}=[0.835,0]^{T}$.

only related to the partitioning of the investigation domain. In other words, only the number of cells to be examined grows exponentially with the system dimensions, i.e. smaller base for the computational complexity. Note that the computation of forward reachable sets using our algorithm has a polynomial complexity $\mathcal{O}\left(n^{5}\right)$ with respect to the number of state variables.

\section{CONCLUSION}

We present an algorithm based on reachability analysis to estimate provable stability regions of an equilibrium point for nonlinear systems. Our results are compared to wellestablished techniques in this area, namely the computation of backward reachable sets starting from a target region using the viscosity solution of a time-dependant HJI PDE, and the optimization of the LF sub-level set using SOS programming. Note that the intention of the presented comparison is not to replace Lyapunov-based techniques or backward reachability computations, but rather to complement them with a more
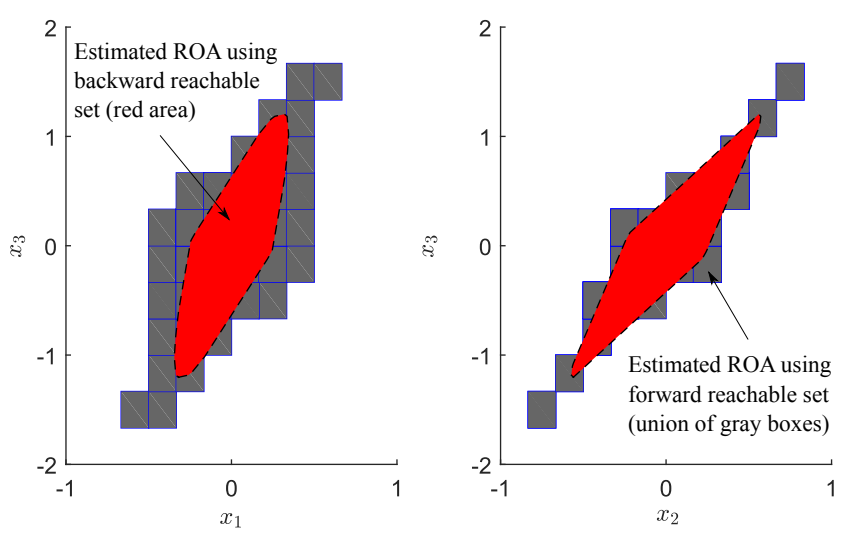

Fig. 6. Projection of the estimation of the ROA of (19) using our proposed algorithm (gray areas), LST (red area with a dotted stroke). The equilibrium point is located at the origin.
TABLE I

COMPARISON OF THE CPU TIME.

\begin{tabular}{cccc}
\hline \multirow{2}{*}{ Model } & \multicolumn{3}{c}{ Computational time } \\
& CORA [29] & SMRSOFT [31] & LST [30] \\
\hline Van-der-Pol & $373.27 \mathrm{~s}$ & $2.63 \mathrm{~s}$ & $476.71 \mathrm{~s}$ \\
2-D example & $72.84 \mathrm{~s}$ & $5.79 \mathrm{~s}$ & $62.39 \mathrm{~s}$ \\
SMIB & $382.71 \mathrm{~s}$ & $7.01 \mathrm{~s}$ & $97.58 \mathrm{~s}$ \\
3-D example & $428.3 \mathrm{~s}$ & unknown & $687.07 \mathrm{~s}$ \\
Pendulum & $571.91 \mathrm{~s}$ & unknown & unknown \\
\hline
\end{tabular}

rigorous stability analysis of nonlinear systems. Each technique has its own share of advantages and disadvantages. For example the Lyapunov approach is superior in terms of CPU time for low-dimensional systems; however it provides a conservative ROA. On the contrary, backward reachability computations provide accurate estimates of the stability region, but the associated memory requirements grow rapidly with the system dimension. Furthermore, it is not a formal method, i.e. it only provides an accurate estimation rather than a provable stability region.

Our proposed approach provides accurate, provable estimates of the ROA and scales moderately with system dimensions. More importantly, the algorithm is not based on Lyapunov stability theory, i.e. it does not use or require the existence of a LF in order to provide a provable ROA. Additionally, the approach is capable of dealing with general non-polynomial systems without redefining them as a polynomial vector.

In the future, we would like to investigate the possibilities of unifying the aforementioned techniques under one framework which only exploits their advantages. Furthermore, to improve accuracy of the estimated ROA, we plan to use polynomial zonotopes, introduced in our previous work [35],

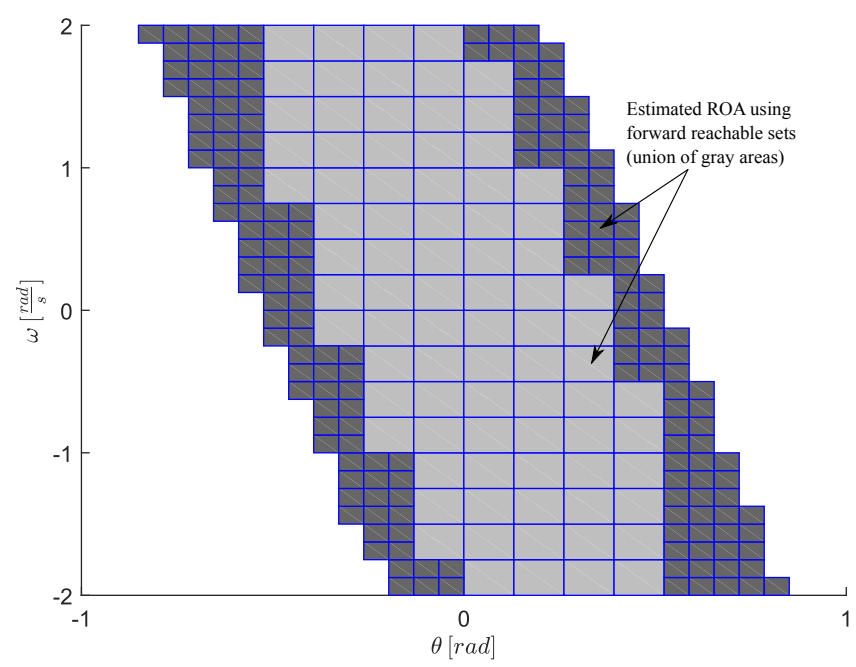

Fig. 7. Projection of the estimation of the ROA of (20) using our proposed algorithm (gray areas). The equilibrium point is located at the origin. 
as set representation for forward reachability computations. Polynomial zonotopes allow one to select larger initial sets, thus reducing the size of the partitioned grid, which in return would substantially reduce the overall computational costs associated with our estimation algorithm. Finally, we intend to combine the proposed approach with a compositional reachability algorithm for transient stability analysis of power systems [36]. This allows one to compute provable stability margins, which are of great importance to transmission system operators to dynamically assess security of a transmission network [33].

\section{ACKNOWLEDGMENT}

Financial support by the German Research Foundation (DFG) is gratefully acknowledged (AL 1185/2-1).

\section{REFERENCES}

[1] E. Glassman, A. L. Desbiens, M. Tobenkin, M. Cutkosky, and R. Tedrake, "Region of attraction estimation for a perching aircraft: A Lyapunov method exploiting barrier certificates," in Proc. of the 2012 IEEE International Conference on Robotics and Automation, 2012, pp. 2235-2242.

[2] T. Lee, M. Leoky, and N. H. McClamroch, "Geometric tracking control of a quadrotor UAV on SE (3)," in Proc. of the 49th IEEE Conference on Decision and Control, 2010, pp. 5420-5425.

[3] J. L. Meza, V. Santibáñez, and R. Campa, "An estimate of the domain of attraction for the PID regulator of manipulators," International Journal of Robotics \& Automation, vol. 22, no. 3, pp. 187-195, 2007.

[4] N. G. Bretas and L. F. Alberto, "Lyapunov function for power systems with transfer conductances: Extension of the invariance principle," IEEE Transactions on Power Systems, vol. 18, no. 2, pp. 769-777, 2003.

[5] M. Anghel, F. Milano, and A. Papachristodoulou, "Algorithmic construction of Lyapunov functions for power system stability analysis," IEEE Transactions on Circuits and Systems I: Regular Papers, vol. 60, no. 9, pp. 2533-2546, 2013.

[6] H. K. Khalil and J. Grizzle, Nonlinear systems. Prentice hall New Jersey, 1996, vol. 3.

[7] I. M. Mitchell, "Comparing forward and backward reachability as tools for safety analysis," in Hybrid systems: computation and control. Springer, 2007, pp. 428-443.

[8] C. Le Guernic, "Reachability analysis of hybrid systems with linear continuous dynamics," Ph.D. dissertation, Université Joseph-FourierGrenoble I, 2009.

[9] M. Althoff, "Reachability analysis and its application to the safety assessment of autonomous cars," Ph.D. dissertation, Technische Universität München, 2010.

[10] A. Vannelli and M. Vidyasagar, "Maximal Lyapunov functions and domains of attraction for autonomous nonlinear systems," Automatica, vol. 21, no. 1, pp. 69-80, 1985.

[11] S. Prajna, A. Papachristodoulou, and F. Wu, "Nonlinear control synthesis by sum of squares optimization: A Lyapunov-based approach," in Proc. of the 5th Asian Control Conference, vol. 1, 2004, pp. 157165.

[12] W. Tan and A. Packard, "Stability region analysis using polynomial and composite polynomial Lyapunov functions and sum-of-squares programming," IEEE Transactions on Automatic Control, vol. 53, no. 2, pp. 565-570, 2008

[13] G. Chesi, Domain of attraction: analysis and control via SOS programming. Springer Science \& Business Media, 2011, vol. 415.

[14] _ - "LMI techniques for optimization over polynomials in control:A survey," IEEE Transactions on Automatic Control, vol. 55, no. 11, pp. 2500-2510, 2010.

[15] A. Majumdar, A. A. Ahmadi, and R. Tedrake, "Control and verification of high-dimensional systems with DSOS and SDSOS programming," in Proc. of the 53rd IEEE Conference on Decision and Control, 2014, pp. $394-401$

[16] D. Han, A. El-Guindy, and M. Althoff, "Estimating the domain of attraction based on the invariance principle," in 55th IEEE Conference on Decision and Control, 2016, pp. 5569-5576.
[17] D. Henrion and M. Korda, "Convex computation of the region of attraction of polynomial control systems," IEEE Transactions on Automatic Control, vol. 59, no. 2, pp. 297-312, 2014.

[18] R. Genesio, M. Tartaglia, and A. Vicino, "On the estimation of asymptotic stability regions: State of the art and new proposals," IEEE Transactions on Automatic Control, vol. 30, no. 8, pp. 747-755, 1985.

[19] L. Jin, R. Kumar, and N. Elia, "Reachability analysis based transient stability design in power systems," International Journal of Electrical Power \& Energy Systems, vol. 32, no. 7, pp. 782-787, 2010.

[20] I. M. Mitchell, A. M. Bayen, and C. J. Tomlin, "A time-dependent Hamilton-Jacobi formulation of reachable sets for continuous dynamic games," IEEE Transactions on Automatic Control, vol. 50, no. 7, pp. 947-957, 2005.

[21] J. K. Scott, "Reachability analysis and deterministic global optimization of differential-algebraic systems," Ph.D. dissertation, Massachusetts Institute of Technology, 2012.

[22] A. Girard, "Reachability of uncertain linear systems using zonotopes," in Hybrid Systems: Computation and Control. Springer, 2005, pp. 291-305.

[23] M. Althoff, O. Stursberg, and M. Buss, "Reachability analysis of nonlinear systems with uncertain parameters using conservative linearization," in Proc. of the 47th IEEE Conference on Decision and Control, 2008, pp. 4042-4048.

[24] A. El-Guindy, D. Han, and M. Althoff, "Formal analysis of drumboiler units to maximize the load-following capabilities of power plants," IEEE Transactions on Power Systems, vol. 31, no. 6, pp. 46914702,2016

[25] A. El-Guindy, K. Schaab, B. Schürmann, O. Stursberg, and M. Althoff, "Formal LPV control for transient stability of power systems," in Proc. of the 2017 IEEE Power and Energy Society General Meeting, 2017, pp. 1-5.

[26] J. H. Gillula, G. M. Hoffmann, H. Huang, M. P. Vitus, and C. J. Tomlin, "Applications of hybrid reachability analysis to robotic aerial vehicles," The International Journal of Robotics Research, vol. 30 , no. 3, pp. 335-354, 2011.

[27] A. Platzer and E. M. Clarke, "The image computation problem in hybrid systems model checking," in Hybrid Systems: Computation and Control. Springer, 2007, pp. 473-486.

[28] M. Berz and G. Hoffstätter, "Computation and application of Taylor polynomials with interval remainder bounds," Reliable Computing, vol. 4, no. 1, pp. 83-97, 1998.

[29] M. Althoff, "An Introduction to CORA 2015," in Proc. of the Workshop on Applied Verification for Continuous and Hybrid Systems, 2015, pp. 120-151, http://www6.in.tum.de/Main/SoftwareCORA.

[30] I. Mitchell. A toolbox of level set methods. [Online]. Available: http://www.cs.ubc.ca/ mitchell/ToolboxLS/

[31] G. Chesi. SMRSOFT: A MATLAB Toolbox for Optimization over Polynomials and Dynamical Systems Study via SOS Programming. [Online]. Available: http://www.eee.hku.hk/ chesi/smrsoft.htm

[32] T.-C. Wang, S. Lall, and M. West, "Polynomial level-set methods for nonlinear dynamical systems analysis," in Proc. of Allerton conference on communication, control and computing, 2005, pp. 640-649.

[33] P. Kundur, N. J. Balu, and M. G. Lauby, Power system stability and control. McGraw-hill New York, 1994, vol. 7.

[34] S. Ratschan and Z. She, "Providing a basin of attraction to a target region of polynomial systems by computation of Lyapunov-like functions," SIAM Journal on Control and Optimization, vol. 48, no. 7, pp. 4377-4394, 2010

[35] M. Althoff, "Reachability analysis of nonlinear systems using conservative polynomialization and non-convex sets," in Hybrid Systems: Computation and Control, 2013, pp. 173-182.

[36] A. El-Guindy, Y. C. Chen, and M. Althoff, "Compositional transient stability analysis of power systems via the computation of reachable sets," in Proc. of the 2017 IEEE Amercian Control Conference, 2017. 